\title{
Training at Moderate Altitude and Under Normobaric Hypoxia Conditions as a Reliable Endurance Development Way
}

\author{
Radchenko $\mathrm{AS}^{1 *}$, Kolesnikov $\mathrm{NV}^{2}$ and Radchenko $\mathrm{AA}^{3}$ \\ ${ }^{1}$ Professor of physical education department, Sc.D., Saint Petersburg University of the Humanities and Social Science \\ (SPbUHSS), Russia \\ ${ }^{2}$ Professor of physical education department, Ph.D., The Russian Presidential Academy of National Economy and Public \\ Administration (RANERPA), Russia
}

${ }^{3}$ Medical equipment engineer, Sport's school, Russia

\begin{abstract}
This article is dedicated to an important problem of contemporary sports - the use of natural and artificial hypoxia in the training process. Briefly outlines the problem origin, the existing training strategies, adaptive reconstructions in the athlete's body, the application of hypoxic training (HT). The prospects of HT relying on various sports and the need for correction (revision) of the existing principles for building the training process are discussed.
\end{abstract}

KEYWORDS: Altitude training; Hypoxia; Performance of athletes

\section{INTRODUCTION}

Study of the biological mechanisms that sense and respond to oxygen levels (normoxia, hypoxia, ischemia) in mammals has promoted a better understanding of the multiple changes in the norm and pathology of physiological processes in humans. Numerous works have opened new opportunities for the elaboration of the treatment methods for such diseases as anemia, cardiovascular and pulmonary diseases, hypertension, stroke, etc. This area of research is recognized as one of the most promising in the treatment development for many illnesses associated with oxygen deficiency in the human body [1].

The empirical coaching approach to the sports training process has revealed significant advantages of training in natural and artificial hypoxia conditions. Since Mexico City, located in high altitude (2250 $\mathrm{m}$ above sea level), was announced (1963) as the venue for the XIX Olympic Games, a focused study of the natural hypoxia effects on a person's physical performance began. Athletes of many sports started to conduct regular multi-week training sessions at moderate and high altitude not only with the purpose of preliminary adaptation to the conditions of natural hypoxia, but also to improve sports results in subsequent competitions at sea level. Gradually, principles of the preparation process were formed, in which hypoxia is used as an additional tool for adaptation to muscle work, especially in cyclic endurance sports. Combinations of using different elevation above sea level with training loads were also substantiated, and technological methods for the impact of

Quick Response Code:

Address for correspondence: Radchenko AS, Professor of physical education department, Sc.D., Saint Petersburg University of the Humanities and Social Science (SPbUHSS), Russia

Received: March 19, 2020 Published: March 31, 2020

How to cite this article: Radchenko AS, Kolesnikov NV, Radchenko AA. Training at Moderate Altitude and Under Normobaric Hypoxia Conditions as a Reliable Endurance Development Way. 2020 - 2(2) OAJBS.ID.000157. DOI: 10.38125/OAJBS.000157 
moderate altitude on the athlete's body were developed in order to improve the results in competitions. Special "hypoxicators" were designed to create an air environment with a low oxygen content, for breathing, both at rest and during muscle work. Even though many years have passed since the well-known generalizing symposium on the problem of altitude training [2], the number of publications around the world on the problem under consideration continues to grow and is difficult to account. Therefore, we cite only a modest part of this flow of scientific periodicals.

We sought to make a brief description of the adaptive adjustments that occur in the athlete's body as a result of exposure to normobaric hypoxia at rest and during muscular work, which are the basis for improving the athlete's sports (professional) level.

\section{Training Strategies}

Different versions of training strategies are used at moderate altitude. The first one is classified as "living high-training high" (LHTH). In this version of the training strategy, athletes live and train in midland at the same altitude above sea level (from $\sim 1,500$ to $\sim 3,000 \mathrm{~m}$ ). The second strategy is "living high-training low" (LHTL). Under the LHTL conditions, athletes live in moderate altitude at $\sim 2500$, but train lower, approximately $\sim 1000-1200 \mathrm{~m}$ above sea level. This strategy was proposed by B Levine \& J StrayGundersen [3]. The third is "living low-training high" (LLTH). In this case, the altitude of the temporary residence and the altitude of the training site are combined in the reverse order. GP Millet et al. [4], analyzing these training strategies, offer additional forms of combining different altitudes and hypoxia levels with the distribution and alternation of training loads. Thus, the trainer can use the cited studies as teaching aids.

In contemporary sports, a constant increase in results in cyclic sports is unthinkable without the use of altitude training and normobaric hypoxia. Further study of the effects of these factors on the athlete's body as an important component of endurance development is an extremely relevant topic for physiological research. Also, in RL Wilber's review [5] the use of devices such as hypoxicators, as well as rooms where an atmosphere with a lower oxygen concentration in the inhaled air created for the athlete during the rest period are discussed. Currently, the use of these devices in sports training around the world has acquired an extraordinary scope because, creating an air flow for breathing with an oxygen concentration from $19.8 \%$ to $9.8 \%$, which corresponds to an altitude from $500 \mathrm{~m}$ to $6000 \mathrm{~m}$ above sea level, they can be used individually. Thus, hypoxic training (HT) was added to the established training strategies, which is used both as a necessary component in conventional training programs for athletes at sea level, and in combination with physical activity at various altitudes.

Adaptive reconstructions in the athlete's body using moderate altitude and HT are described in several reviews [4-8]. Adjustments in the athlete's body occur along the entire path of transport and utilization of oxygen from the alveoli of the lungs to the muscles, heart, central nervous system, and other organs and tissues. Further, we will address only some of the questions that relate to adaptive changes in the body during oxygen transfer. We limit ourselves to the discussions of adaptive reconstructions in the system of oxygen transport and utilization in working muscles in athletes.

\section{Changes in the Oxygen Transport Function of the Blood}

It is known that during the implementation of multi-week training programs in the middle altitude, quantitative changes in the blood composition are observed. Due to an increase in the concentration of erythropoietin, the number of red blood cells, reticulocytes, and hemoglobin ( $\mathrm{Hb}$ ) mass is increasing. The hematocrit, soluble transferrin receptor increases, and the saturation of arterial $\mathrm{Hb}$ with oxygen $\left(\mathrm{SpO}_{2}\right)$ and others gradually increase [9-11]. If the regular repetition of high intensity and duration exercises in hypoxia conditions is combined with breathing air with low oxygen content at rest, this gives an additional effect to an increase in the quantitative blood indices [4]. The study of the adaptive response of erythropoiesis as a training result under hypoxic conditions remains the subject of study. For detailed familiarization with the problem, you can refer to the reviews $[12,13]$. And yet, to single out the quantitative changes in the blood composition as signs of adaptation to the altitude only partially reflects the reconstructions in the athlete's body to hypoxic environment conditions $[14,15]$. Non-hematological mechanisms of adaptation to altitude and hypoxia also play a significant role. At the same time, exercise effectiveness [16-19] that based on the improvement of microcirculation and mitochondrial efficiency [4] is improved.

One of the adaptation mechanisms is the expansion of blood vessels and micro vessels during severe hypoxia. In this regard, let us pay attention to the dual role of an erythrocyte in providing oxygen to tissues, which has been studied in the past more than two decades [20,21-24]. It is known that red blood cells of vertebrate's service tissues in two ways. First, erythrocytes provide the opportunity for adequate $\mathrm{O}_{2}$ transport between the respiratory surface of the lungs and tissues through a high concentration of $\mathrm{Hb}$ in them, corresponding allosteric relations between the ligand-binding sites of $\mathrm{Hb}$, and a regulated intracellular chemical environment, which allows one to sharply tune the relationship of $\mathrm{Hb}$ to $\mathrm{O}_{2}$. Secondly, red blood cells can sense the $\mathrm{O}_{2}$ request by tissues due to the degree of deoxygenation when moving through micro vessels secreting vasodilating formations which expand the blood flow in hypoxic tissues. This last function is important in triggering $\mathrm{O}_{2}$ tissue delivery in connection with a local $\mathrm{O}_{2}$ demand [24]. The second function of red blood cells may be the main one because the delivery of $\mathrm{O}_{2}$ to tissues at a great distance depends on the systemic blood flow. The picture is created that erythrocytes are not only a transport that carries a large amount of $\mathrm{O}_{2}$ into the tissues, but they also control the effectors of local blood flow. This allows contributing to hypoxic vasodilation, in a mechanism guaranteed to quickly trigger local $\mathrm{O}_{2}$ delivery in muscles by $\mathrm{O}_{2}$ demands $[25,24]$. The oxygen advancement through the membranes of erythrocytes, the walls of small and the smallest arteries and capillaries into tissue cells of active organs occurs along the $\mathrm{O}_{2}$ concentration gradient, forming an oxygen tensions field [26]. Inside muscle fibers (MF), diffusion of $\mathrm{O}_{2}$ to mitochondria is also provided by the difference in the partial tension of $\mathrm{O}_{2}$.

After the end of an intense and prolonged exercise during the period of acute recovery of the body, there is an intensification in the reconstructions of many mechanisms involving hemoproteins. So, due to an increase in hepcidin concentration in blood serum during the period of acute recovery after exercise with an intensity of 60$90 \%$ of $\dot{V} O_{2}$ max, the ferroportin transport channel is destroyed (disrupted), the ability of macrophages to process "damaged" iron decreases, and its availability decreases.

The hepcidin fraction circulation increases during early recovery after exercise ( $\sim 3$ hours). It remains unknown how acute muscular exercise can alter hepcidin expression [27]. Hypoxia is one of the hepcidin synthesis regulators. It weakens its expression and increases the expression of ferroportin [27-28]. During this 
period reliable availability of iron is especially required to maintain $\mathrm{Hb}$ mass that can be a serious problem in athletes $[16,31,32]$ which must be considered in the training process. Recommended iron therapy should be based on a preliminary analysis of ferritin and transferrin levels in blood serum. In the example of long-distance runners it was shown that at a low concentration of ferritin $(<35$ $\mathrm{mg} / \mathrm{L}$ ) and transferrin saturation $<20 \%$ the iron therapy (duration of iron therapy is 6 weeks) is more effective than in runners with suboptimal (<65 mg/L) ferritin concentration. Herewith, the intravenous use of iron-containing medicines ensures iron availability more efficiently (ferritin grows faster), and significantly increases the duration of work to exhaustion at $\dot{V} O_{2}$ max compared to the oral use of appropriate agents [33]. It's necessary to add that over the course of a day iron is best absorbed in the morning, immediately after training despite an increase of hepcidin level in blood [34].

\section{Intracellular Mechanisms of $\mathrm{O}_{2}$ Transport and Utilization}

It is important to consider that adaptive changes involving hemoproteins occur along the entire path of $\mathrm{O}_{2}$ transport and utilization in the organism. In addition to $\mathrm{O}_{2}$ diffusion, when hypoxia increases, myoglobin $(\mathrm{Mb})$ is connected, which facilitates the diffusion of $\mathrm{O}_{2}$ to mitochondria. The portion ratio of the $\mathrm{O}_{2}$ diffusion and $\mathrm{Mb}$-facilitating $\mathrm{O}_{2}$ diffusion mechanism in the total oxygen flow to mitochondria in the sarcoplasm varies in accordance with the change of hypoxia level and muscle work power as it approaches $\dot{V} O_{2} \max [35,36,15]$. After the termination of an intense and prolonged muscular work, there is an increase of mitochondria production in MF. Their total oxidizing surface increases, especially when exposed to hypoxia [37-40].

It has been shown that at the final stage of $\mathrm{O}_{2}$ delivery and utilization inside the MF with a very long respiratory delay, which is carried out by the elite level divers, there is equal control of $\mathrm{Mb}$ activity, but lower consumption of $\mathrm{O}_{2}$ by mitochondria in divers in comparison with the selected control group [41]. This denotes into a decreased level of muscular performance in divers. At the same time, as $\dot{V} \mathrm{O}_{2}$ max is reached, when all the mechanisms that ensure the maximum transport of $\mathrm{O}_{2}$ to mitochondria are mobilized, a more detailed study of the behavior of the Mb-facilitating diffusion of $\mathrm{O}_{2}$ mechanism remains a problem [41]. At that, adaptive reconstructions of mitochondria are quite understandable, and the development of the necessary research methods is encouraging [42].

Thus, the rise of capillary network density, mobilization of erythropoiesis, an increase in the overall oxidative surface of mitochondria, and a possible strengthening of the Mb-facilitating diffusion of $\mathrm{O}_{2}$ mechanism (which remains to be shown in the experiment) increase the delivery and utilization of $\mathrm{O}_{2}$ in $\mathrm{MF}$ as the load approaches $\dot{V} O_{2}$ max. Long-term adaptive changes are additionally supported by hypoxic conditions of muscle work. There is a sufficient amount of specialized knowledge to improve the training process.

\section{Training with the Use of Hypoxia}

The implementation of long-term training programs in moderate altitude and the long-term use (multiple during the year-round training cycle, as well as long-term application) of normobaric HT intentionally creates an athlete advantages over regular training in normoxic conditions.

In connection with this statement, we present data from studies using normobaric hypoxia in the training process for several micro-cycles. Using the example of long-distance runners it was demonstrated that the additional inclusion of training on a treadmill with an intensity of the ventilation threshold 2 level duration of 24-40 minutes, and breathing during exercise with mixture containing $14.5 \%$ oxygen ( $\sim 3000 \mathrm{~m}$ above sea level) within a regular workout program, enhances the metabolic stimulus to the muscles. So, despite a slight increase of $\dot{V O}_{2}$ max in the subjects, a significant rise in the duration of the run at $\mathrm{VO}_{2} \max$ to exhaustion was revealed, by almost $42 \%$. The authors showed that for this contingent of athletes, the performed training series had a targeted effect on the aerobic energy supply of muscles [43-45].

In addition to these works, there are several publications substantiating the use of HT in sports games [46,47], swimming [48-50]. GP Millet and O Girard [6] cite a number of works that explain the methods of HT in such sports as cycling, country ski racing, football, rugby [51-55]. Details can be found in metaanalyzes [11,30].

\section{Possibilities of HT Employment in Other Sports Not Listed Above}

It is known that fatigue during cyclic work approaching $\dot{V} \mathrm{O}_{2}$ max has both a central and peripheral component. At the same time, supraspinal commands (motor control) support the preservation of muscle work power at the limit of intensity and duration of physical load. There are details in Amann \& Calbet [56].

Since the increased motor activity of a person during sports for a long period of his life is clearly manifested in morpho-functional reconstructions of his brain [57], it can be confidently stated that HT can significantly improve performance (adaptability) not peripheral (muscle) energy production mechanisms only and the central apparatus of motor control that are specifical in each of sport. It is necessary to emphasize that degree mobilization of the central mechanisms for voluntary movement control different sports are different significantly too.

For example, a 1,500-m runner or a pursuit-racer on a cycle track, working on $\dot{V} O_{2}$ max for $\sim 4$ minutes, solves the relatively simple task of his movement control. Supraspinal commands primarily provide the highest possible muscle performance. At the same time interval, the figure skater performs a large number of complicated coordinated actions with the maximum speed-power motor qualities mobilization. Against the background of central and local fatigue increasing induced by maximal muscular work, the figure skaters' CNS is utmost mobilizing the vestibular stability and accuracy of movements [53,54].

The HT technique allows through hypoxicator to affect different levels of motor control and their energy supply. Thus, in conditions of hard hypoxia at rest when the inhalation $\mathrm{O}_{2}$ fraction $\left(\mathrm{F}_{1} \mathrm{O}_{2}\right)$ is $\sim 0.1$, the athlete undergoes a restructuring of the blood flow in CNS and heart. Under conditions of moderate hypoxia $\left(\mathrm{F}_{\mathrm{I}} \mathrm{O}_{2} \sim 0.14\right.$ 0.15 ) during muscle work, the reorganization of the blood flow in CNS and heart is added by the reorganization of the blood flow and other adaptive reconstructions in the muscles $[55,56]$.

Exercises to balance maintaining under hard hypoxia for several minutes are more significantly mobilize the central and spinal postural control mechanisms, i.e., with repeated use significantly improve sensorimotor coordination, and cyclic exercises with individually selected severe and moderate hypoxia more effectively trigger off the muscle adaptation. Thus, the use of HT by figure skaters will enlarge the level of their aerobic performance and vestibular stability (VS) without increasing the total time spent 
on general physical training. It is important to emphasize that the figure skater who has a higher level of aerobic performance is much faster recovery despite the fact that he performs high-speed power muscular work in training. The growth of aerobic performance allows him to mastermind more volume of speed-power work in the total training program [57].

Even though figure skating selects children with high by nature VS, in the monograph by AN Mishin \& VA Shapiro [58] gives experimentally justified diagnostics of VS and methods of individual selection of rotation loads to improving this basic motor quality in elite level of figure skaters. Mild and moderate hypoxia conditions (selected individually $\mathrm{F}_{1} \mathrm{O}_{2} \sim 0.17-0.15$ ) during rotation can significantly enhance the effectiveness of the vestibular resistance training device.

The CNS blood circulation strengthening under hard hypoxia conditions and relative muscle rest at aiming in shooting should contribute to additional mobilization of the sensorimotor cortex and all subcortical structures of the corresponding fields in the visual and motor (fingers) analyzers. Combinations of various training means (for example, the use of electronic aim simulators at standing on the platform for equilibrium maintenance) are improved the aiming under hypoxia conditions not only in biathlonists, but also in shooters from any type of weapon. These ways allow significantly increase the training volume with an emphasis on aiming, because mobilization of cortical and subcortical centers, which provide targeted action, is important for improving and consolidating a stable motor skill "for accuracy".

The experience of HT in beach soccer teams [59] shows that the use of HT can significantly increase the level of aerobic performance in traditional footballers. The specificity of beach soccer is running by sand, which strengthens the static component in muscle contraction. The combination of HT with game actions on loose soil, compared with running on a lawn or firm (elastic) artificial lawn, significantly enhances local muscle endurance. The combination of the targeted effects of hypoxia on CNS and leg muscles in special exercises significantly enhances aerobic endurance. The combination can maintain a high level of aerobic endurance individually throughout the game season with strict monitoring of the athlete's functional state.

In conclusion, it's necessary to emphasize that aerobic performance in any sport can significantly enhance by the use of HT. The athlete has an advantage in his main type of activity due to faster recovery between intense and prolonged training and competition. The conditions are created for the implementation of higher quality special preparing in a particular sport. It remains up to the coach to apply HT in the training program of his sport, learn to combine the usual training loads with hypoxia exposure. Based on our own experience of working with coaches of various sports specializations, it is important to add, that there is no increase in the total volume of training work. However, it will take great efforts to thoroughly revise the "well-beaten" training plans that have been practiced for years and familiar (conventional) views on the training process.

\section{REFERENCES}

1. Hurst JH (2016) William Kaelin, Peter Ratcliffe and Gregg Semenza receive the 2016 Albert Lasker Basic Medical Research Award. J Clin Invest 126(10): 3628-3638.

2. Med Sci Sports Exerc 2007 39(9): 1587-1631.

3. Levine BD (1997) Living high-training low: effect of moderate-altitude acclimatization with lowaltitude training on performance. J Appl Physiol 83: 102-112.

4. Millet GP, Roels B, Schmitt L, Woorons X, Richalet JP (2010) Combining hypoxic methods for peak performance. Sports Med 40(1): 1-25.

5. Wilber RL (2007) Application of altitude/hypoxic training by elite athletes. Med Sci Sports Exerc 39: 1610-1624.

6. Radchenko AS (2016) Nitric oxide and hypoxia at adaptation to muscular work (brief review). Reviews on clinical pharmacology and drug therapy 14(1): 78-88.

7. Millet GP, Girard O (2017) Editorial: High-intensity exercise in hypoxia: Beneficial aspects and potential drawbacks. Front Physiol 8: 1017.

8. Viscor G, Torrella JR, Corral L, Ricart A, Javierre C, et al. (2018) Physiological and biological responses to short-term intermittent hypobaric hypoxia exposure: from sports and mountain medicine to new biomedical applications. Front Physiol 9: 814.

9. Stray-Gundersen J, Chapman RF, Levine BD (2001) Living high-training low altitude training improves sea level performance in male and female elite runners. J Appl Physiol 91(3):1113-1120.

10. Schmitt L, Millet G, Robach P, Nicolet G, Brugniaux JV, et al. (2006) Influence of living high-training low on aerobic performance and economy of work in elite athletes. Eur J Appl Physiol 97(5): 627-636.

11. Rusko HK, Tikkanen H, Paavolainen L, Hamalainen I, Kalliokoski K, et al. (1999) Effect of living in hypoxia and training in normoxia on sea level $\dot{\mathrm{V}} \mathrm{O}_{2}$ max and red cell mass. Med Sci Sports Exerc 31: S86.

12. Park HY, Hwang H, Park J, Lee S, Lim K (2016) The effects of altitude/ hypoxic training on oxygen delivery capacity of the blood and aerobic exercise capacity in elite athletes-a meta-analysis. J Exerc Nutrition Biochem 20(1):15-22

13. Płoszczyca K, Langfort J, Czuba M (2018) The effects of altitude training on erythropoietic response and hematological variables in adult athletes: a narrative review. Front Physiol 9: 375.

14.Gore CJ, Hopkins WG (2005) Counterpoint: positive effects of intermittent hypoxia (live high: train low) on exercise performance are not mediated primarily by augmented red cell volume. J Appl Physiol 99(5): 2055-2057.

15. Lin PC, Kreutzer U, Thomas J (2007) Myoglobin translational diffusion in rat myocardium and its Implication on intra cellular oxygen transport. J Physiol 578: 595-603.

16. Gore CJ, Hahn AG, Aughey RJ, Martin DT, Ashenden MJ, et al. (2001) Live high: train low increases muscle buffer capacity and submaximal cycling efficiency. Acta Physiol Scand 173(3): 275-286.

17. Gore CJ, Clark SA, Saunders PU (2007) Nonhematological mechanisms of improved sea-level performance after hypoxic exposure. Med Sci Sports Exerc 39(9): 1600-1609.

18. Saunders PU, Telford RD, Pyne DB, Cunningham RB, Gore CJ, et al. (2004) Improved running economy in elite runners after 20 days of simulated moderate-altitude exposure. J Appl Physiol 96: 931-937.

19. Schmitt L, Millet G, Robach P (2006) Influence of "living high-training low" on aerobic performance and economy of work in elite athletes. Eur J Appl Physiol 97: 627-636.

20. Ellsworth ML, Forrester T, Ellis CG, Dietrich HH (1995) The erythrocyte as a regulator of vascular tone. Amer J Physiol 269: H2155-H2161.

21. González-Alonso J (2012) ATP as a mediator of erythrocyte-dependent regulation of skeletal muscle blood flow and oxygen delivery in humans. J Physiol 590(20): 5001-5013.

22. González-Alonso J, Olsen DB, Saltin B (2002) Erythrocyte and the regulation of human skeletal muscle blood flow and oxygen delivery: role of circulating ATP. Circ Res 91(11):1046-1055.

23. González-Alonso J, Mortensen SP, Dawson EA, Secher NH, Damsgaard R (2006) Erythrocytes and the regulation of human skeletal muscle blood flow and oxygen delivery: role of erythrocyte count and oxygenation state of haemoglobin. J Physiol 572(1): 295305. 
24. Jensen FB (2009) The dual roles of red blood cells in tissue oxygen delivery: oxygen carriers and regulators of local blood flow. J Exp Biol 212: 3387-3393

25. Gladwin MT, Raat NJ, Shiva S, Dezfulian C, Hogg N, et al. (2006) Nitrite as a vascular endocrine nitric oxide reservoir that contributes to hypoxic signaling, cytoprotection, and vasodilation. Amer J Physiol Heart Circ Physiol 291(5): H2026-H2035.

26. Ivanov KP (1993) The principles of energetics in organism: Theoretical and practical aspects. Vol. 2. Biological oxidations and its providing with oxygen.

27. Domínguez R, Sánchez-Oliver AJ, Mata-Ordoñez F, Feria-Madueño A, Grimaldi-Puyana M, et al. (2018) Effects of an acute exercise bout on serum hepcidin levels. Nutrients 10(2): 209.

28. Nicolas G, Chauvet C, Viatte L, Danan JL, Bigard X, et al. (2002) The gene encoding the iron regulatory peptide hepcidin is regulated by anaemia, hypoxia and inflammation. J Clin Investig 110(7): 1037-1044.

29. Talbot NP, Lakhal S, Smith TG, Privat C, Nickol AH, et al. (2012) Regulation of hepcidin expression at high altitude. Blood 119(3): 857-860.

30. Goetze O, Schmitt J, Spliethoff K, Igor T, Guenter W, et al. (2013) Adaptation of iron transport and metabolism to acute high-altitude hypoxia in mountaineers. Hepatology 58: 2153-2162.

31. Hinton PS (2014) Iron and the endurance athlete. Appl Physiol Nutr Metab 39: 1012-1018.

32. Brocherie F, Girard O, Faiss R, Millet GP (2007) Effects of repeated-sprint training in hypoxia on sea-level performance: a meta-analysis. Sports Med 47(8): 1651-1660.

33. Garvican LA, Saunders PU, Cardoso T, Macdougall IC, Lobigs LM, et al. (2014) Intravenous iron supplementation in distance runners with low or suboptimal ferritin. Med Sci Sports Exerc 46(2): 376-385

34. McCormick R, Moretti D, McKay AKA (2019) The impact of morning versus afternoon exercise on iron absorption in athletes. Med Sci Sports Exerc 51(10): 2147-2155.

35. Gros G, Wittenberg BA, Jue T (2010) Myoglobin's old and new clothes: from molecular structure to function in living cells. J Exp Biol 213: 27132725 .

36. Kanatous SB, Mammen PPA (2010) Regulation of myoglobin expression J Exper Biol 213: 2741-2747.

37. Hood DA (2001) Invited review: contractile activity-induced mitochondrial biogenesis in skeletal muscle. J Appl Physiol 90(3): 11371157.

38. Jacobs RA, Lundby C (2013) Mitochondria express enhanced quality as well as quantity in association with aerobic fitness across recreationally active individuals up to elite athletes. J Appl Physiol 114(3): 344-350.

39. Jacobs RA, Boushel R, Wright-Paradis C, Calbet JA, Robach P, et al. (2013) Mithohondrial function in human skeletal muscle following highaltitude exposure. Exp Physiol 98(1): 245-255.

40. Lundby C, Jacobs RA (2016) Adaptations of skeletal muscle mitochondria to exercise training. Exp Physiol 101(1): 17-22.

41. Kjeld T, Stride N, Gudiksen A, Hansen EG, Arendrup HC, et al. (2018) Oxygen conserving mitochondrial adaptations in the skeletal muscles of breath hold divers. PLoS ONE 13(9): e0201401.

42. Doerrier C, Garcia-Souza LF, Krumschnabel G, Wohlfarter Y, Mészáros AT, et al. (2018) High-resolution fluorespirometry and oxphos protocols for human cells, permeabilized fibers from small biopsies of muscle, and isolated mitochondria. Methods Mol Biol 1782: 31-70.

43. Ponsot E, Dufour SP, Zoll J, Doutrelau S, N'Guessan B, et al. (2006) Exercise training in normobaric hypoxia in endurance runners. II. Improvement of mitochondrial properties in skeletal muscle. J Appl Physiol 100(4):1249-1257.

44. Dufour SP, Ponsot E, Zoll J, Doutreleau S, Lonsdorfer-Wolf E, et al (2006) Exercise training in normobaric hypoxia in endurance runners. I. Improvement in aerobic performance capacity. J Appl Physiol $100(4): 1238-1248$

45. Zoll J, Ponsot E, Dufour S, Doutreleau S, Ventura-Clapier R, et al. (2005) Exercise training in normobaric hypoxia in endurance runners. Muscular adjustments of selected gene transcripts. J Appl Physiol 100(4): 1258 -1266 .

46. Billaut F, Gore CJ, Aughey RJ (2012) Enhancing team-sport athlete performance: is altitude training relevant? Sports Med 42(9):751-767.

47. Brocherie F, Millet GP, Hauser A (2015) Live high-train low and high hypoxic training improves team-sport performance. Med Sci Sports Exerc 47: 2140-2149.

48. Rodríguez FA, Iglesias X, Feriche B, Calderón-Soto C, Chaverri D, et al. (2015) Altitude training in elite swimmers for sea level performance. Med Sci Sports Exerc 47(9): 1965-1978.

49. Park HY, Lim K (2017) Effects of hypoxic training versus normoxic training on exercise performance in competitive swimmers. J Sports Sci Med 16(4): 480-488.

50. Czuba M, Wilk R, Karpiński J, Chalimoniuk M, Zajac A, et al. (2017) Intermittent hypoxic training improves anaerobic performance in competitive swimmers when implemented into a direct competition mesocycle. PLoS ONE 12(8): e0180380.

51. Faiss R, Girard 0, Millet GP (2013) Advancing hypoxic training in team sports: from intermittent hypoxic training to repeated sprint training in hypoxia. Br J Sports Med 47(1): i45-i50.

52. Faiss R, Léger B, Vesin JM, Fournier PE, Eggel Y, et al. (2013) Significant molecular and systemic adaptations after repeated sprint training in hypoxia. PLoS ONE 8(2): e56522.

53. Faiss R, Willis S, Born DP, Sperlich B, Vesin JM, et al. (2015) Repeated double-poling sprint training in hypoxia by competitive cross-country skiers. Med Sci Sports Exerc 47: 809-817.

54. Galvin HM, Cooke K, Sumners DP, Mileva KN, Bowtell JL (2013) Repeated sprint training in normobaric hypoxia. Br J Sports Med 47(1): i74-i79.

55. Gatterer H, Philippe M, Menz V, Mosbach F, Faulhaber M, et al. (20014) Shuttle-run sprint training in hypoxia for youth elite soccer players: a pilot study. J Sports Sci Med 13: 731-735.

56. Amann M, Calbet JAL (2008) Convective oxygen transport and fatigue. Appl Physiol 104(3): 861-870.

57. Batouli SAH, Saba V (2017) At least eighty percent of brain grey matter is modifiable by physical activity: A review study. Behavioral brain research 332: 205-217.

58. Mishin AN, Shapiro VA (2015) Figure skating as a space flight. St Petersburg.

59. Radchenko AS, Elisarov AB, Radchenko AA (2019) The special endurance development in beach soccer players. Physical Culture and Youth Health: XV All-Russian Scientific and Practical Conference, Petersburg. 